Gut and Liver, Vol. 11, No. 5, September 2017, pp. 628-634

\title{
Characteristics of Primary and Metachronous Gastric Cancers Discovered after Helicobacter pylori Eradication: A Multicenter Propensity Score- Matched Study
}

Yuji Maehata ${ }^{1}$, Shotaro Nakamura ${ }^{2}$, Motohiro Esaki ${ }^{1}$, Fumie Ikeda ${ }^{1}$, Tomohiko Moriyama ${ }^{1}$, Risa Hida ${ }^{3}$, Ema Washio ${ }^{1}$, Junji Umeno ${ }^{1}$, Minako Hirahashi ${ }^{3}$, Takanari Kitazono ${ }^{1}$, and Takayuki Matsumoto ${ }^{2}$

${ }^{1}$ Department of Medicine and Clinical Science, Graduate School of Medical Sciences, Kyushu University, Fukuoka, ${ }^{2}$ Division of Gastroenterology, Department of Internal Medicine, School of Medicine, Iwate Medical University, Morioka, and ${ }^{3}$ Department of Anatomic Pathology, Graduate School of Medical Sciences, Kyushu University, Fukuoka, Japan

Background/Aims: Gastric cancers develop even after successful Helicobacter pylori eradication. We aimed to clarify the characteristics of early gastric cancers discovered after H. pylori eradication. Methods: A total of 1,053 patients with early gastric cancer treated by endoscopic submucosal dissection were included. After matching the propensity score, we retrospectively investigated the clinicopathological features of 192 patients, including 96 patients who had undergone successful $H$. pylori eradication (Hp-eradicated group) and 96 patients who had active $H$. pylori infection ( $H p$-positive group). Results: In the $\mathrm{Hp}$-eradicated group, early gastric cancers were discovered 1 to 15 years (median, 4.1 years) after $H$. pylori eradication. Compared with $H p$-positive patients, $\mathrm{Hp}$-eradicated patients showed a more frequently depressed configuration ( $81 \%$ vs $53 \%$, respectively, $p<0.0001$ ) and a higher trend toward submucosal invasion ( $18 \%$ vs $8 \%$, respectively, $p=0.051$ ). A multivariable analysis revealed the macroscopic depressed type to be characteristics of early gastric cancers after $\mathrm{H}$. pylori eradication. Among patients in the $\mathrm{Hp}$-eradicated group, metachronous cancers showed less frequent depressed lesions ( $68 \%$ vs $84 \%$, respectively, $\mathrm{p}=0.049$ ) and smaller tumor sizes (median, $11 \mathrm{~mm}$ vs 14 $\mathrm{mm}$, respectively, $\mathrm{p}=0.014$ ) than primary cancers. Conclusions: Early gastric cancers after $H$. pylori eradication are characterized by a depressed configuration. Careful followup endoscopies are necessary after $H$. pylori eradication. (Gut Liver 2017;11:628-634)

Key Words: Helicobacter pylori eradication; Early gastric cancer; Endoscopic submucosal dissection; Metachronous gastric cancer; Depressed configuration

\section{INTRODUCTION}

Gastric cancer is the fifth most common cancer and the third leading cause of cancer death in the world. ${ }^{1}$ It has been clarified that the development of gastric cancer is mostly caused by Helicobacter pylori infection..$^{2-4}$ In 2008, a Japanese randomized trial showed that $H$. pylori eradication contributed to a significant reduction in the incidence of metachronous gastric cancer (MGC) after endoscopic resection of early primary gastric cancer (PGC). ${ }^{5}$ In contrast, our previous retrospective study indicated that $H$. pylori eradication did not significantly reduce the incidence of MGC during a follow-up period of up to 11 years. ${ }^{6}$ A metaanalysis of 13 clinical studies from Japan and Korea showed that $H$. pylori eradication after endoscopic resection of early PGC significantly reduced the incidence of MGC. ${ }^{7-11}$ However, these studies also have revealed that gastric cancers certainly occur even after successful $H$. pylori eradication. It thus seems to be important to recognize the clinical features of those cancers discovered after $H$. pylori eradication for their appropriate diagnosis and treatment. To date, the characteristics of gastric cancers, which developed after $H$. pylori eradication, have not been clarified.

In the present study, we have conducted a multicenter propensity score-matched study to clarify the characteristics of early gastric cancers discovered after $H$. pylori eradication.

\footnotetext{
Correspondence to: Yuji Maehata

Department of Medicine and Clinical Science, Graduate School of Medical Sciences, Kyushu University, Maidashi 3-1-1, Higashi-ku, Fukuoka 8128582, Japan

Tel: +81-92-642-5261, Fax: +81-92-642-5273, E-mail: ymaehata@intmed2.med.kyushu-u.ac.jp

Received on July 19, 2016. Revised on September 8, 2016. Accepted on November 9, 2016. Published online April 11, 2017

pISSN 1976-2283 eISSN 2005-1212 https://doi.org/10.5009/gnl16357

(a) This is an Open Access article distributed under the terms of the Creative Commons Attribution Non-Commercial License (http://creativecommons.org/licenses/by-nc/4.0) which permits unrestricted non-commercial use, distribution, and reproduction in any medium, provided the original work is properly cited.
} 


\section{MATERIALS AND METHODS}

\section{Study subjects}

This was a multicenter, retrospective, and propensity scorematched study from Kyushu University Hospital and other 18 hospitals in Fukuoka, Yamaguchi, and Ehime, Japan. All these hospitals have participated the Kyushu University multicenter endoscopic submucosal dissection database (KYU-MED) study. This database was approved by the Institutional Review Board of Kyushu University Hospital and has been registered in the University Hospital Medical Information Network Clinical Trials Registry (UMIN-CTR) as number UMIN000009190. Written informed consent was obtained from each patient included in the study.

Between June 2003 and October 2014, a total of 2,153 patients with early gastric cancer who were treated by endoscopic submucosal dissection (ESD) ${ }^{12}$ at the participating hospitals were registered in KYU-MED study. Of these, 670 patients were not investigated for their $H$. pylori status, and 418 patients showed negative results for examinations for $H$. pylori infection without definite history of $H$. pylori eradication therapy. Of these patients, 58 patients had been assessed by both ${ }^{13} \mathrm{C}$-urea breath test and IgG serology, 39 by ${ }^{13} \mathrm{C}$-urea breath test, and two patients by both stool $H$. pylori antigen test and serology, while 251 patients had been tested only by serology, 48 only by rapid urease test, and the remaining 20 patients only by histology. In other 12 patients, gastric cancer was resected within 1 year after H. pylori eradication. These 1,100 patients were excluded. The remaining 1,053 patients were further divided into the following two groups: (1) 117 patients who had undergone successful H. pylori eradication more than 1 year before, as confirmed by a negative result of ${ }^{13} \mathrm{C}$-urea breath test or serology at the time of ESD (Hp-eradicated group); (2) 936 patients who had an active $H$. pylori infection, as determined by the rapid urease test, ${ }^{13} \mathrm{C}-$ urea breath test, stool $H$. pylori antigen test, histology or serology ( $\mathrm{Hp}$-positive group). After analyzing the baseline clinical characteristics of 1,053 patients, 1:1 propensity score-matching was performed to minimize any selection bias. ${ }^{13}$ Finally, a total of 192 matched patients, with 96 patients in each group, were included in the analysis (Fig. 1).

\section{Clinicopathological assessment of gastric cancers}

In this study, PGC was defined as a gastric carcinoma that developed in a patient who had no previous history of gastric cancer. MGC was defined as a new carcinoma that developed in other areas at least 1 year after the endoscopic resection of the initial gastric cancer. In patients with MGC, the initial early gastric cancer had been treated by ESD or endoscopic mucosal resection in another hospital. All endoscopic examinations and ESDs were performed by the specialized endoscopists who had a license for Board Certification of Japan Gastroenterological Endoscopy Society in 18 participated hospitals.

The histopathological assessment of the resected specimens was performed by the senior pathologist in each hospital. In 44 patients who had more than one carcinoma at the time of ESD, we evaluated only one carcinoma of which the depth of invasion and/or size was greater than the others. Among these 44 patients, we chose the carcinoma invading the submucosa in spite of the smaller size in three patients. Location, macroscopic type, and histological findings of gastric cancers were classified according to the Japanese classification and Paris classification of gastric carcinoma. ${ }^{14,15}$ Namely, the location was classified by dividing stomach into three equal sections: upper, middle, and lower. Macroscopic type was classified either as elevated (type 0-I, IIa, and IIa+IIc) or as depressed type (type 0-IIb, IIc, IIc+IIa, IIc+III, and III). Histopathologically, well and/or moderately differentiated adenocarcinomas were regarded as differentiated type, while poorly differentiated adenocarcinoma and/or signet-

2,153 Patients with early gastric cancer treated by ESD

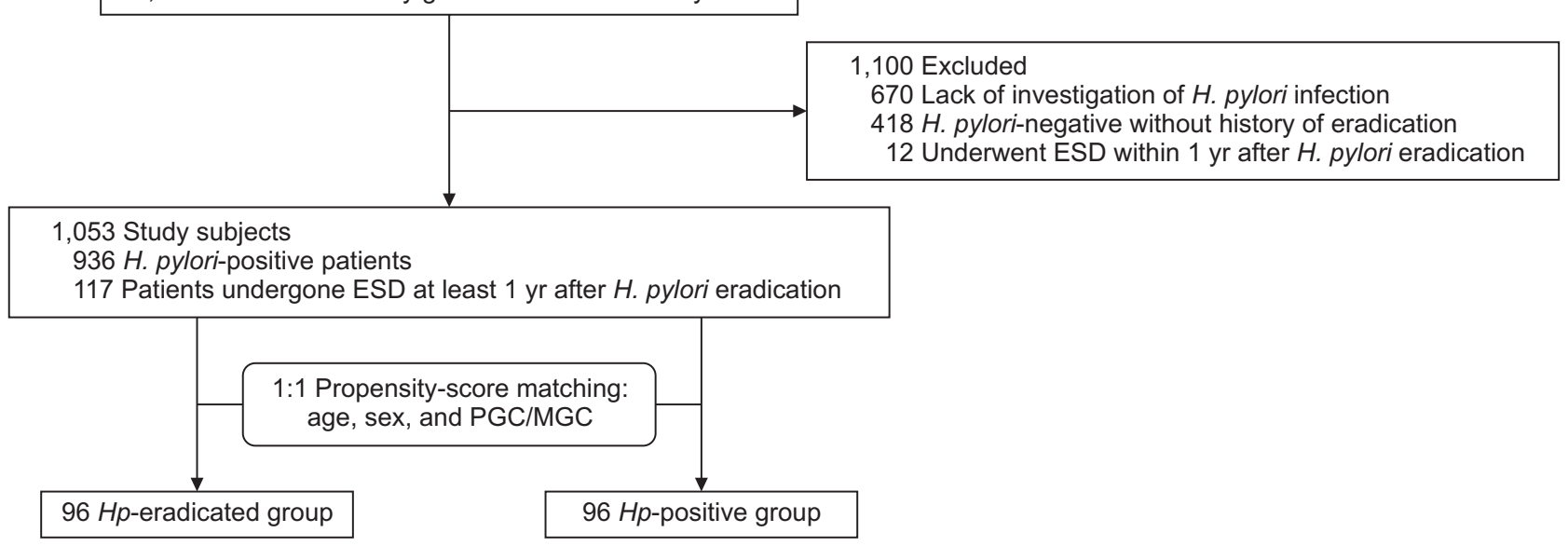

Fig. 1. Flow of the study subjects.

ESD, endoscopic submucosal dissection; PGC, primary gastric cancer; MGC, metachronous gastric cancer; Hp, Helicobacter pylori. 
ring cell carcinoma were as undifferentiated type. Presence or absence of ulcerations with or without scars, submucosal invasion, lymphatic invasion and venous invasion were also assessed with the resected specimens. When massive invasion to the submucosa and/or lymphovascular invasion by carcinoma was observed, an additional gastrectomy with regional lymph node dissection was recommended to the patient.

\section{Statistical analysis}

Two of the authors (Y.M. and F.I.) performed all the statistical analyses. F.I. is an expert for the statistics. We used propensity score-matching analysis to adjust significant differences in the baseline clinical characteristics of patients and to reduce the influence of possible confounding factors. Propensity scores were calculated using a logistic regression model and variables included in the model were age, sex, and PGC/MGC (Fig. 1). After propensity scores were estimated, one-to-one nearest-neighbor matching without replacement was performed with a caliper of width equal to 0.2 of the standard deviation of the logit of the propensity score. ${ }^{13}$ Before and after propensity score-matching, we compared the clinicopathological characteristics of these enrolled patients between $\mathrm{Hp}$-positive group and $\mathrm{Hp}$-eradicated group. Among $\mathrm{Hp}$-eradicated patients, the clinicopathological characteristics were also compared between PGC and MGC. The parametric data were expressed as the medians (range). The numerical data were compared using the Mann-Whitney U test, and the categorical data were compared by the Fisher exact probability test or the chi-square test. In order to identify the independent characteristics of early gastric cancer after $H$. pylori eradication, the variables that were assumed to influence the characteristics of early gastric cancer with p-values less than 0.2 were put into a multivariable analysis using logistic regression

Table 1. Comparison of Clinicopathological Characteristics between Hp-Eradicated and Hp-Positive Groups before and after Propensity-Score Matching

\begin{tabular}{|c|c|c|c|c|c|c|}
\hline \multirow[b]{2}{*}{ Characteristic } & \multicolumn{3}{|c|}{ All patients $(\mathrm{n}=1,053)$} & \multicolumn{3}{|c|}{ Propensity-matched patients $(n=192)$} \\
\hline & $\begin{array}{c}H p \text {-eradicated } \\
(\mathrm{n}=117)\end{array}$ & $\begin{array}{l}\text { Hp-positive } \\
(\mathrm{n}=936)\end{array}$ & p-value & $\begin{array}{l}\text { Hp-eradicated } \\
(\mathrm{n}=96)\end{array}$ & $\begin{array}{c}H p \text {-positive } \\
(\mathrm{n}=96)\end{array}$ & p-value \\
\hline Sex & & & 0.075 & & & 0.855 \\
\hline Male & $95(81)$ & $691(74)$ & & $77(80)$ & $78(81)$ & \\
\hline Female & $22(19)$ & $245(26)$ & & $19(20)$ & $18(19)$ & \\
\hline Age, yr & $71(46-90)$ & $71(30-96)$ & 0.397 & $72(46-90)$ & $72(47-92)$ & 0.886 \\
\hline Primary or metachronous cancers & & & $<0.0001$ & & & 1.000 \\
\hline Primary gastric cancer & $67(57)$ & 907 (97) & & $67(70)$ & $67(70)$ & \\
\hline Metachronous gastric cancer & $50(43)$ & $29(3)$ & & $29(30)$ & $29(30)$ & \\
\hline Location & & & 0.358 & & & 0.982 \\
\hline Upper & $24(21)$ & $148(16)$ & & $21(22)$ & $22(23)$ & \\
\hline Middle & $45(38)$ & $352(38)$ & & $36(37)$ & $35(36)$ & \\
\hline Lower & $48(41)$ & $436(46)$ & & $39(41)$ & $39(41)$ & \\
\hline Macroscopic type & & & 0.001 & & & $<0.0001$ \\
\hline Elevated type & $27(23)$ & 354 (38) & & $18(19)$ & $45(47)$ & \\
\hline Depressed type & $90(77)$ & $582(62)$ & & $78(81)$ & $51(53)$ & \\
\hline Size (diameter), mm & $13(2-75)$ & $14(1-100)$ & 0.052 & $13(2-75)$ & $14(2-60)$ & 0.820 \\
\hline Histology & & & 0.473 & & & 0.721 \\
\hline Differentiated type & $114(97)$ & 892 (95) & & $93(97)$ & $91(95)$ & \\
\hline Undifferentiated type & $3(3)$ & $44(5)$ & & $3(3)$ & $5(5)$ & \\
\hline Depth of invasion & & & 0.190 & & & 0.051 \\
\hline Mucosa & $97(83)$ & $818(87)$ & & $79(82)$ & $88(92)$ & \\
\hline Submucosa & $20(17)$ & $118(13)$ & & $17(18)$ & $8(8)$ & \\
\hline Ulceration or scar & $11(9.4)$ & $66(7.1)$ & 0.374 & $9(9.4)$ & $7(7.3)$ & 0.601 \\
\hline Lymphatic invasion & $7(6.0)$ & $25(2.7)$ & 0.076 & $6(6.3)$ & $3(3.1)$ & 0.497 \\
\hline Venous invasion & $2(1.7)$ & $12(1.3)$ & 0.663 & $1(1)$ & 0 & 1.000 \\
\hline Adjunctive gastrectomy after ESD & $11(9.4)$ & $66(7.1)$ & 0.374 & $10(10)$ & $5(5.2)$ & 0.175 \\
\hline
\end{tabular}

Data are presented as number (\%) or median (range).

Hp, Helicobacter pylori; ESD, endoscopic submucosal dissection. 
model. A p-value of $<0.05$ was considered to be statistically significant for each test. All analyses were performed with the JMP Pro 12.2.0 software (SAS Institute, Cary, NC, USA).

\section{RESULTS}

\section{Comparison between $\mathrm{Hp}$-eradicated group and Hp-positive group}

In $\mathrm{Hp}$-eradicated patients, early gastric cancers were discovered 1 to 15 years (median, 4.1 years) after $H$. pylori eradication. Table 1 compares the clinicopathological features of patients between $\mathrm{Hp}$-eradicated group and $\mathrm{Hp}$-positive group before and after propensity score-matching. Before propensity scorematching, male patients were tended to be more frequent in $H p$-eradicated group (81\%) than in $\mathrm{Hp}$-positive group (74\%; $\mathrm{p}=0.075)$. MGC was more frequent in $\mathrm{Hp}$-eradicated group (50/117 patients, 43\%) than in Hp-positive group (29/936 patients, $3 \% ; \mathrm{p}<0.0001)$. Depressed configuration was more frequent in $\mathrm{Hp}$-eradicated group (77\%) than in $\mathrm{Hp}$-positive group $(62 \% ; p=0.001)$. There were trends towards smaller size of can- cer (median, $13 \mathrm{~mm}$ vs $14 \mathrm{~mm}$; $\mathrm{p}=0.052$ ) and high prevalence of lymphatic invasion ( $6 \%$ vs $2.7 \%$; $=0.076$ ) in Hp-eradicated group than in $\mathrm{Hp}$-positive group.

After propensity score-matching, 67 patients with PGC and 29 patients with MGC were included in each group $(\mathrm{p}=1.000)$. Macroscopically, depressed type was more frequent in $\mathrm{Hp}$-erad-

Table 2. Results of a Multivariate Logistic Regression Analysis for the Characteristics of Early Gastric Cancers Discovered after Helicobacter pylori Eradication in Propensity-Matched Patients

\begin{tabular}{lccc}
\hline & OR & $95 \% \mathrm{CI}$ & $\mathrm{p}$-value \\
\hline $\begin{array}{l}\text { Macroscopic type } \\
\quad \text { Depressed type vs elevated type }\end{array}$ & 3.80 & $2.00-7.49$ & $<0.0001$ \\
$\begin{array}{l}\text { Depth of invasion } \\
\quad \text { Submucosa vs mucosa }\end{array}$ & 1.85 & $0.65-5.69$ & 0.252 \\
$\begin{array}{l}\text { Adjunctive gastrectomy after ESD } \\
\quad \text { Necessary vs unnecessary }\end{array}$ & 1.56 & $0.40-6.43$ & 0.518 \\
\hline
\end{tabular}

OR, odds ratio; CI, confidence interval; ESD, endoscopic submucosal dissection.

Table 3. Comparison of the Clinicopathological Characteristics among All Patients with PGC and Those with MGC Discovered after Helicobacter pylori Eradication

\begin{tabular}{|c|c|c|c|}
\hline & PGC (n=67) & MGC $(n=50)$ & $\mathrm{p}$-value \\
\hline Sex & & & 0.847 \\
\hline Male & $54(81)$ & $41(82)$ & \\
\hline Female & $13(19)$ & $9(18)$ & \\
\hline Age, yr & $69(46-85)$ & $74(56-90)$ & 0.003 \\
\hline Time after $H$. pylori eradication, yr & $4.9(1.0-15.0)$ & $3.8(1.0-14.0)$ & 0.263 \\
\hline Time after latest negative endoscopy, yr & $1.9(0.5-10.0)$ & $1.0(0.5-2.0)$ & $<0.0001$ \\
\hline Location & & & 0.627 \\
\hline Upper & $15(23)$ & $9(18)$ & \\
\hline Middle & $27(40)$ & $18(36)$ & \\
\hline Lower & $25(37)$ & $23(46)$ & \\
\hline Macroscopic type & & & 0.049 \\
\hline Elevated type & $11(16)$ & $16(32)$ & \\
\hline Depressed type & $56(84)$ & $34(68)$ & \\
\hline Size (diameter), mm & $14(3-75)$ & $11(2-43)$ & 0.014 \\
\hline Histology & & & 1.000 \\
\hline Differentiated type & $65(97)$ & $49(98)$ & \\
\hline Undifferentiated type & $2(3)$ & $1(2)$ & \\
\hline Depth of invasion & & & 0.473 \\
\hline Mucosa & $57(85)$ & $40(80)$ & \\
\hline Submucosa & $10(15)$ & $10(20)$ & \\
\hline Ulceration or scar & $5(7.5)$ & $6(12)$ & 0.408 \\
\hline Lymphatic invasion & $2(3)$ & $5(10)$ & 0.136 \\
\hline Venous invasion & 0 & $2(4)$ & 0.281 \\
\hline Adjunctive gastrectomy after ESD & $4(6)$ & $7(14)$ & 0.201 \\
\hline
\end{tabular}

Data are presented as number (\%) or median (range).

PGC, primary gastric cancer; MGC, metachronous gastric cancer; ESD, endoscopic submucosal dissection. 
icated group (81\%) than in Hp-positive group (53\%; $\mathrm{p}<0.0001)$. Submucosal invasion of carcinoma tended to be more frequent in $\mathrm{Hp}$-eradicated group (18\%) than in $\mathrm{Hp}$-positive group (8\%; $\mathrm{p}=0.051$ ). Other factors, including sex, age, location, size, histologic type, ulceration, lymphatic and venous invasion, and adjunctive gastrectomy after ESD, did not differ between two groups.

\section{Independent clinicopathological characteristics of early gastric cancer after $\boldsymbol{H}$. pylori eradication}

As shown in Table 2, our multivariable logistic regression analysis determined that macroscopic depressed type (odds ratio, 3.80; 95\% confidence interval, 2.00 to 7.49 ; $p<0.0001$ ) was the sole independent characteristics of early gastric cancer after $H$. pylori eradication in propensity-matched patients. Other factors, including depth of invasion and adjunctive gastrectomy after ESD, did not significantly associate with cases discovered after $H$. pylori eradication.

\section{Comparison between PGC and MGC discovered after H. pylori eradication}

Table 3 compares the clinicopathological features between patients with PGC and those with MGC in Hp-eradicated group before propensity score-matching. Patients with MGC were older than those with PGC (median, 74 years vs 69 years; $p=0.003$ ). The median time interval between $H$. pylori eradication and ESD of cancers did not differ between patients with PGC (4.9 years) and those with MGC (3.8 years). By contrast, time interval between the latest negative endoscopy and ESD of cancers was longer in patients with PGC than in those with MGC (median, 1.9 years vs 1.0 years; $\mathrm{p}<0.0001)$. Macroscopically, depressed lesion was more frequent in PGC (84\%) than in MGC (68\%; $p=0.049)$. The size of MGC was smaller than that of PGC (median, $11 \mathrm{~mm}$ vs $14 \mathrm{~mm} ; \mathrm{p}=0.014)$. There were trends towards high prevalence of lymphatic invasion (10\% vs 3\%), venous invasion ( $4 \%$ vs $0 \%$ ), and adjunctive gastrectomy (14\% vs 6\%) in MGC than in PGC. However, these differences were not statistical significant. Other factors, including sex, location, and histologic type did not differ between two groups.

\section{DISCUSSION}

In the present study, we confirmed that gastric cancers including both PGCs and MGCs developed during a long-term period of up to 15 years (median, 4 years) after $H$. pylori eradiation. Compared to $H$. pylori-positive cancers, early gastric cancer after $H$. pylori eradication had more frequent depressed configuration and a higher trend of submucosal invasion. Our multivariable analysis revealed macroscopic depressed type to be independent characteristics of early gastric cancer after $H$. pylori eradication.

To date, there have been reported several studies investigat- ing the characteristics of gastric cancers discovered after $H$. pylori eradication. ${ }^{16-20}$ In a follow-up study, Kamada et al. ${ }^{16}$ found that gastric cancers discovered after $H$. pylori eradication were characterized by noncardiac location, small size $\leq 20 \mathrm{~mm}$, macroscopically ulcerative type, and histologic intestinal type. In a pathological study by Yamamoto et al., ${ }^{19}$ gastric cancers detected after $H$. pylori eradication showed smaller size, more frequent macroscopic depressed type, and lower Ki-67 labeling index than $H$. pylori-positive gastric cancers. They speculated that improvement of gastric acid secretion induced after $H$. pylori eradication may prevent the elevated growth in the surface of gastric cancers. ${ }^{19}$ Interestingly, Ito et al. ${ }^{21}$ indicated that about half of the elevated type of gastric carcinoma and adenoma became flattened only one month after $H$. pylori eradication. They speculated that $H$. pylori eradication might have directly inhibited the upward (expansive) growth of the gastric tumor. It should be noted that the number of subjects with gastric cancers discovered after the eradication in our current study ( $n=96)$ was much larger than in these previous studies ( $\mathrm{n}=18$ to 47 ). ${ }^{16-20} \mathrm{In}$ addition, this was a propensity score-matched study. We thus consider our results to possess higher reliability than those of previous studies.

In the present study, MGC was more frequent in $\mathrm{Hp}$-eradicated group (43\%) than in Hp-positive group (3\%) before propensity score-matching. Since the publication of Japanese randomized trial in $2008,^{5}$ almost all patients with first early gastric cancer treated by endoscopic resection in Japan have undergone $H$. pylori eradication therapy. ${ }^{22,23}$ Thus, absolute numbers of $H$. pylori-positive patients with MGC seem to have decreased in these recent years. It has been demonstrated that severe mucosal atrophy with high risk of subsequent development of MGCs still remains in most of those patients with a history of removal of early gastric cancer, even after successful eradication of $H$. pylori. ${ }^{6,24}$ Therefore, careful follow-up endoscopy should be applied especially in patients with early gastric cancer treated by endoscopic resection and $H$. pylori eradication.

It has been considered that $H$. pylori eradication may inhibit the progression of gastric cancer to some extent. ${ }^{19,25}$ A Korean randomized trial showed that all the 10 MGCs in eradicated group were confined to the mucosa, while six of $14(43 \%)$ MGCs in $H$. pylori-positive group invaded submucosa or muscularis propria. ${ }^{8}$ In our present matched study, however, submucosal invasion of carcinoma tended to be more frequent in $\mathrm{Hp}$-eradicated group (18\%) than in $\mathrm{Hp}$-positive group (8\%; $\mathrm{p}=0.051$ ). In a Japanese randomized trial, the prevalence of submucosal invasion in MGCs did not differ between $\mathrm{Hp}$-eradicated group (11\%) and $H$. pylori-positive group (4\%). ${ }^{5}$ It thus seems controversial as to whether invasive MGCs increase or decrease after $H$. pylori eradication. Several studies have revealed that normal columnar epithelia and "gastritis-like" appearances are often observed on the surface of early gastric cancers after $H$. pylori eradication. ${ }^{21,26}$ We thus speculate that such cancers tend 
to show indistinct endoscopic appearances and to take longer time to be discovered in some cases, and as a consequence, they invade the submucosa during the prolonged period.

In $\mathrm{Hp}$-eradicated group, tumor size was larger depressed lesion was more frequent in PGC than in MGC (Table 3). This observation was probably associated with the fact that time after latest negative endoscopy was longer in patients with PGC (1.9 years) than in those with MGC (1.0 years). However, higher trends of lymphatic invasion, venous invasion, and adjunctive gastrectomy in MGC than in PGC seem to suggest that MGC after $H$. pylori eradication may haven more aggressive behavior.

Our present study has several limitations. First, our study subjects were limited to patients with early gastric cancer treated by ESD, and patients with advanced gastric cancers or those obviously invading the submucosa were excluded. Thus, our results might not represent the characteristics of all the gastric cancers discovered after $H$. pylori eradication. Second, many subjects were excluded because of lack of investigation for $H$. pylori infection $(\mathrm{n}=670)$ or negative results for $H$. pylori $(\mathrm{n}=418)$. Among $H$. pylori-negative patients $(n=418), 319$ patients were examined only by the rapid urease test, histology, or serology. Therefore, false-negative patients who were not confirmed by ${ }^{13} \mathrm{C}$-urea breath test or stool $H$. pylori antigen test would be included in these patients. Third, we had no data of background mucosal atrophy in each patient and we obtained the data of latest negative endoscopy only for $\mathrm{Hp}$-eradicated group. In addition, retrospective nature of the study might have introduced certain selection biases.

In conclusion, our multicenter propensity score-matched study revealed that early gastric cancers after $H$. pylori eradication are characterized by depressed configuration and higher trend of submucosal invasion. The long-term risk for the development of gastric cancer exists even in patients who have achieved successful $H$. pylori eradication. We should perform follow-up endoscopy carefully after $H$. pylori eradication, with special attentions to depressed type gastric cancer. Further investigations of larger number of patients with much longer follow-up periods are therefore still required to clarify the clinicopathological characteristics of gastric cancers that develop after $H$. pylori eradication.

\section{CONFLICTS OF INTEREST}

No potential conflict of interest relevant to this article was reported.

\section{ACKNOWLEDGEMENTS}

This study was undertaken with the following hospitals in Fukuoka, Yamaguchi and Ehime Prefecture, Japan: Kyushu University Hospital, Kimura Hospital, Kyushu Central Hospital, Chihaya Hospital, Hamanomachi Hospital, Hakujyuji Hospital,
Fukuoka Sanno Hospital, Fukuoka Red Cross Hospital, Onga Hospital, Kama Red Cross Hospital, Kyushu Hospital, Kokura Medical Center, Steel Memorial Yawata Hospital, Saiseikai Yahata General Hospital, Fukuoka Yutaka Central Hospital, Moji Ekisaikai Hospital, Shimonoseki City Hospital, Yamaguchi Red Cross Hospital, and Matsuyama Red Cross Hospital.

\section{REFERENCES}

1. Fock KM. Review article: the epidemiology and prevention of gastric cancer. Aliment Pharmacol Ther 2014;40:250-260.

2. Yamagata H, Kiyohara Y, Aoyagi K, et al. Impact of Helicobacter pylori infection on gastric cancer incidence in a general Japanese population: the Hisayama study. Arch Intern Med 2000;160:19621968.

3. Uemura N, Okamoto S, Yamamoto S, et al. Helicobacter pylori infection and the development of gastric cancer. N Engl J Med 2001;345:784-789.

4. International Agency for Research on Cancer, World Health Organization. Schistosomes, liver flukes and Helicobacter pylori. IARC Monogr Eval Carcinog Risks Hum 1994;61:177-241.

5. Fukase K, Kato M, Kikuchi S, et al. Effect of eradication of Helicobacter pylori on incidence of metachronous gastric carcinoma after endoscopic resection of early gastric cancer: an open-label, randomized controlled trial. Lancet 2008;372:392-397.

6. Maehata Y, Nakamura S, Fujisawa K, et al. Long-term effect of Helicobacter pylori eradication on the development of metachronous gastric cancer after endoscopic resection of early gastric cancer. Gastrointest Endosc 2012;75:39-46.

7. Seo JY, Lee DH, Cho Y, et al. Eradication of Helicobacter pylori reduces metachronous gastric cancer after endoscopic resection of early gastric cancer. Hepatogastroenterology 2013;60:776-780.

8. Choi J, Kim SG, Yoon H, et al. Eradication of Helicobacter pylori after endoscopic resection of gastric tumors does not reduce incidence of metachronous gastric carcinoma. Clin Gastroenterol Hepatol 2014;12:793-800.e1.

9. Bae SE, Jung HY, Kang J, et al. Effect of Helicobacter pylori eradication on metachronous recurrence after endoscopic resection of gastric neoplasm. Am J Gastroenterol 2014;109:60-67.

10. Kwon YH, Heo J, Lee HS, Cho CM, Jeon SW. Failure of Helicobacter pylori eradication and age are independent risk factors for recurrent neoplasia after endoscopic resection of early gastric cancer in 283 patients. Aliment Pharmacol Ther 2014;39:609-618.

11. Yoon SB, Park JM, Lim CH, Cho YK, Choi MG. Effect of Helicobacter pylori eradication on metachronous gastric cancer after endoscopic resection of gastric tumors: a meta-analysis. Helicobacter 2014;19:243-248.

12. Gotoda T, Yamamoto H, Soetikno RM. Endoscopic submucosal dissection of early gastric cancer. J Gastroenterol 2006;41:929942.

13. Austin PC. Statistical criteria for selecting the optimal number of untreated subjects matched to each treated subject when using 
many-to-one matching on the propensity score. Am J Epidemiol 2010;172:1092-1097.

14. Japanese Gastric Cancer Association. Japanese classification of gastric carcinoma: 3rd English edition. Gastric Cancer 2011;14:101-112.

15. Participants in the Paris Workshop. The Paris endoscopic classification of superficial neoplastic lesions: esophagus, stomach, and colon: November 30 to December 1, 2002. Gastrointest Endosc 2003;58(6 Suppl):S3-S43.

16. Kamada T, Hata J, Sugiu K, et al. Clinical features of gastric cancer discovered after successful eradication of Helicobacter pylori: results from a 9-year prospective follow-up study in Japan. Aliment Pharmacol Ther 2005;21:1121-1126.

17. Mabe K, Takahashi M, Oizumi H, et al. Does Helicobacter pylori eradication therapy for peptic ulcer prevent gastric cancer? World J Gastroenterol 2009;15:4290-4297.

18. Take S, Mizuno M, Ishiki K, et al. The long-term risk of gastric cancer after the successful eradication of Helicobacter pylori. J Gastroenterol 2011;46:318-324.

19. Yamamoto K, Kato M, Takahashi M, et al. Clinicopathological analysis of early-stage gastric cancers detected after successful eradication of Helicobacter pylori. Helicobacter 2011;16:210-216.

20. Matsuo T, Ito M, Tatsugami M, et al. Gastric cancer development after Helicobacter pylori eradication therapy: a new form of gas- tric neoplasia. Digestion 2012;85:61-67.

21. Ito M, Tanaka S, Takata S, et al. Morphological changes in human gastric tumours after eradication therapy of Helicobacter pylori in a short-term follow-up. Aliment Pharmacol Ther 2005;21:559566.

22. Asaka M, Kato M, Takahashi S, et al. Guidelines for the management of Helicobacter pylori infection in Japan: 2009 revised edition. Helicobacter 2010;15:1-20.

23. Kim SG, Jung HK, Lee HL, et al. Guidelines for the diagnosis and treatment of Helicobacter pylori infection in Korea, 2013 revised edition. J Gastroenterol Hepatol 2014;29:1371-1386.

24. Mori G, Nakajima T, Asada K, et al. Incidence of and risk factors for metachronous gastric cancer after endoscopic resection and successful Helicobacter pylori eradication: results of a large-scale, multicenter cohort study in Japan. Gastric Cancer 2016;19:911918.

25. Kato M, Asaka M, Ono S, et al. Eradication of Helicobacter pylori for primary gastric cancer and secondary gastric cancer after endoscopic mucosal resection. J Gastroenterol 2007;42 Suppl 17:1620.

26. Kobayashi M, Hashimoto S, Nishikura K, et al. Magnifying narrow-band imaging of surface maturation in early differentiatedtype gastric cancers after Helicobacter pylori eradication. J Gastroenterol 2013;48:1332-1342. 